\title{
Sex Is Related to Overall Survival in Patients with Esophageal Squamous Cell Carcinoma: A Population-Based Study
}

\author{
Yaoxia Li ${ }^{1}$, Na Wang ${ }^{2}$, Zhuorui Zhao ${ }^{1}$, Jiale Wang ${ }^{1}$, Jun Lyu $^{3}$, Qinyang Wu ${ }^{1}$, Qiqi Ke ${ }^{1}$, \\ Qiaohong Yang, ${ }^{1, *}$
}

${ }^{1}$ School of Nursing, Jinan University, Guangzhou, China

${ }^{2}$ School of Nursing and Health, Henan University, Kaifeng, China

${ }^{3}$ Department of Clinical Research, The First Affiliated Hospital of Jinan University, Guangzhou, China

Email address:

yqiaohong@163.com (Qiaohong Yang)

${ }^{*}$ Corresponding author

\section{To cite this article:}

Yaoxia Li, Na Wang, Zhuorui Zhao, Jiale Wang, Jun Lyu, Qinyang Wu, Qiqi Ke, Qiaohong Yang. Sex Is Related to Overall Survival in Patients with Esophageal Squamous Cell Carcinoma: A Population-Based Study. American Journal of Biomedical and Life Sciences.

Vol. 9, No. 1, 2021, pp. 43-52. doi: 10.11648/j.ajbls.20210901.16

Received: January 8, 2021; Accepted: January 18, 2021; Published: January 25, 2021

\begin{abstract}
Esophageal cancer is one of the cancers that seriously threaten the lives and health of patients around the world. Squamous cell carcinoma accounts for a higher proportion of esophageal cancer cases. At the same time, the number of esophageal squamous cell carcinoma (ESCC) patients also has an increasing trend year by year. Therefore, it is important to identify the prognostic factors of ESCC to improve the survival and prognosis of patients. As an important sociodemographic factor, gender has an important influence on the occurrence, development and prognosis of certain diseases. However, it has not been clear from existing studies whether gender affects the prognosis of ESCC patients. The aim of this study was to determine the effect of sex on overall survival (OS) in patients with ESCC. Methods: This study analyzed 6890 patients with ESCC diagnosed from 2007 to 2015 who were identified in the SEER (Surveillance, Epidemiology, and End Results) database. Kaplan-Meier method and Cox Proportional Hazards model were used to conduct survival analysis and regression analysis to evaluate the association between gender and OS. Results: We found that the OS differed with sex in patients with ESCC, with the 5 -year OS rate being higher in females (19.2\%) than in males (12.9\%). A Cox multivariate analysis showed that sex was an independent prognostic factor for ESCC, with the OS being significantly better in female patients than in male patients $(P<0.001)$. Subgroup analyses showed that sex affected the survival rate of patients with American Joint Committee on Cancer tumor, node, and metastasis stages I $(P=0.013)$, II $(P<0.001)$, III $(P=0.014)$, and IV $(P<0.001)$. Conclusion: Our results suggest that the OS of patients with ESCC is significantly better in females than males. Therefore, more attention should be paid to the prognosis of male patients with ESCC, with prospective interventions and health education applied in order to improve their survival outcomes.
\end{abstract}

Keywords: Sex, Esophageal Squamous Cell Carcinoma, Survival, Prognosis, Database

\section{Introduction}

Esophageal cancer is the sixth most common cause of cancer-related deaths around the world [1]. It is estimated that there are 17,650 new cases of esophageal cancer in the United States annually, with 16,080 patients with esophageal cancer dying each year [2]. Squamous cell carcinoma and adenocarcinoma are the two most common types of esophageal cancer [3]. The epidemiology of esophageal cancer in developed countries has changed recently, with adenocarcinoma surpassing squamous cell carcinoma to become the dominant type of esophageal cancer $[4,5]$. 
However, squamous cell carcinoma still accounts for nearly $30 \%$ of cases of esophageal cancer [6]. Although the prognosis of patients with esophageal squamous cell carcinoma (ESCC) has improved due to advances in radiotherapy, chemotherapy and surgical techniques [7-9], the overall 5-year survival rate is still less than $20 \%[10,11]$. It is [11] therefore particularly important to identify the prognostic factors for ESCC in order to improve the survival rate of patients and the accuracy of prognoses.

As an important sociodemographics factor, sex exerts independent effects on the occurrence and development of diseases $[12,13]$. Some diseases are similar between the sexes, whereas others have large sex-related differences [14, 15]. Understanding the characteristics and effects of sex in disease development is helpful for improving prevention strategies, treatments, and prognoses [12-15]. Therefore, the potential importance of sex as a demographic factor should not be neglected in patients with ESCC, especially as previous studies have shown that being female is associated with better survival outcomes in liver cancer, bladder cancer, renal pelvis cancer, and ureteral cancer [16, 17]. In addition, previous studies have shown that surgery, tumor staging, race, and other factors affect the overall survival (OS) of patients with ESCC [18]. However, the effect of sex on the prognosis of ESCC patients remains unclear.

The purpose of this research was to illustrate the effects of sex on the prognosis of ESCC patients. The research examined the impact of sex on overall survival by analyzing demographic data obtained from the Surveillance, Epidemiology, and End Results (SEER) database.

\section{Material and Methods}

\subsection{Patient Population and Study Design}

The data analyzed in this population-based survey came from the SEER program of the National Cancer Institute of the United States. The SEER database is based on data from local registries in 19 regions of the country, covering about $28 \%$ of the population of the United States, and contains information on all event cases such as general demographic information as well as information on tumor characteristics, treatment, and survival $[19,20]$. The population covered by the SEER database is highly representative of the total population of the United States [21].

We identified 6890 patients with ESCC in the SEER database who had been diagnosed between 2007 and 2015 . The following SEER variables were used to identify patients: "Site Recode ICD-O-3/WHO 2008 classification" (esophagus) and "Histology recode-broad groupings" (histology codes: 8050-8089). The study only included patients with well-documented follow-up data; those with imperfect data registration or a first primary cancer that was not ESCC were excluded.

This study included insurance status as a variable, but since insurance status was first reported by the SEER program in 2007, we included only patients who were first diagnosed between 2007 and 2015 and for whom there were complete insurance records in the database. Based on the information in the SEER database, we classified the insurance status into insured, Medicaid, and uninsured. The marital status is divided into married (common-law marriage) and unmarried (including widowed, divorced, separated, unmarried, and single patients, and those with a domestic partner who they were not married to).

\subsection{Study Variables}

We retrieved the following data from the SEER: sex (female or male), age [three groups based on the following optimal cutoff values identified according to the X-tile program (Yale University version 3.6.1): $\leq 71,72-79$, or $\geq 80$ years], race (white, black, or other), year of diagnosis (2007-2009, 2010 2012, or 2013-2015), tumor grade (I, well differentiated; II, moderately differentiated; III, poorly differentiated; or IV, undifferentiated), sixth edition of the American Joint Committee on Cancer tumor, node, and metastasis (AJCC TNM) staging system (stage I, II, III, or IV), surgery (yes or no), tumor size $(<2,2-4$, or $>4 \mathrm{~cm}$, or unknown), marital status (married or unmarried), and insurance status (insured, Medicaid, or uninsured). Data on follow-up time and life status were also included. The above variables were analyzed statistically, and variables for which $P<0.1$ in the univariate analyses were included in a Cox multivariate analysis.

\subsection{Statistical Analyses}

To facilitate comparisons of the characteristics of patients, mean \pm standard deviation values were used to represent continuous variables. Continuous variables that conformed to a nonnormal distribution were compared using Student's $t$ test, while Pearson's $\chi^{2}$ test was used to compare classified variables. OS was the primary end point, and was defined as the time from the diagnosis of ESCC to death. OS was calculated using the Kaplan-Meier graphic method, and the differences between groups in these diagrams were compared using logarithmic rank test. Cox regression models were used to determine which of the following variables were related to OS: sex, race, age, year of diagnosis, tumor grade, AJCC TNM stage, surgery status, tumor size, insurance status, and marital status. The optimal cutoff points for age were determined using minimum probability value from log-rank $\chi^{2}$ statistics with the aid of the X-tile program, which resulted in age groups of $\leq 71,72-79$, and $\geq 80$ years being included in the Cox multivariate analysis.

All of the statistical tests were bilateral, and differences were considered to be statistically significant when $P<0.05$. SPSS (version 20.0, SPSS, Chicago, Illinois, USA) was used for all of the statistical analyses.

\section{Results}

\subsection{Baseline Characteristics}

Table 1 lists the baseline characteristics of the 6890 analyzed patients stratified by sex: the 2417 (35.08\%) females 
and $4473(64.92 \%)$ males had median ages of 69 and 66 years, respectively. Compared to the females, more of the males were married $(52.60 \%$ vs. $39.70 \%)$ and white $(70.70 \%$ vs. $62.00 \%$ ), whereas more of the female patients had received surgery $(20.60 \%$ vs. $17.00 \%)$. There were significant sex-related differences in age $(P<0.001)$, race $(P<0.001)$, tumor grade $(P<0.01)$, AJCC TNM stage $(P<0.001)$, surgery status $(P<0.001)$, insurance status $(P<0.001)$, and marital status $(P<0.001)$.

Table 1. Patient and tumor characteristics of patients with Esophageal squamous cell carcinoma, SEER 2007-2015.

\begin{tabular}{|c|c|c|c|c|}
\hline Characteristic & Total & Female & Male & P-value \\
\hline Patients, n (\%) & $6890(100.00)$ & $2417(35.08)$ & $4473(64.92)$ & \\
\hline Age, mean \pm SD & & $68.75 \pm 11.58$ & $66.44 \pm 10.78$ & $<0.001$ \\
\hline Race (\%) & & & & $<0.001$ \\
\hline White & $4484(65.10)$ & $1709(70.70)$ & $2775(62.00)$ & \\
\hline Black & $1722(25.00)$ & $528(21.80)$ & $1194(26.70)$ & \\
\hline Other & $684(9.90)$ & $180(7.40)$ & $504(11.30)$ & \\
\hline Year $(\%)$ & & & & 0.884 \\
\hline $2007-2009$ & $2324(33.70)$ & $809(33.50)$ & $1515(33.90)$ & \\
\hline $2010-2012$ & $2314(33.60)$ & $809(33.50)$ & $1505(33.60)$ & \\
\hline $2013-2015$ & $2252(32.70)$ & $799(33.00)$ & $1453(32.50)$ & \\
\hline Grade $(\%)$ & & & & 0.001 \\
\hline I & $409(5.90)$ & $145(6.00)$ & $264(5.90)$ & \\
\hline II & $3399(49.30)$ & $1272(52.60)$ & $2127(47.60)$ & \\
\hline III & $3020(43.80)$ & $979(40.50)$ & $2041(45.60)$ & \\
\hline IV & $62(0.90)$ & $21(0.90)$ & $41(0.90)$ & \\
\hline AJCC TNM stage (\%) & & & & $<0.001$ \\
\hline I & $1166(16.90)$ & $474(19.60)$ & $692(15.50)$ & \\
\hline II & $1720(25.00)$ & $678(28.10)$ & $1042(23.30)$ & \\
\hline III & $1853(26.90)$ & $648(26.80)$ & $1205(26.90)$ & \\
\hline IV & $2151(31.20)$ & $617(25.50)$ & $1534(34.30)$ & \\
\hline Surgery $(\%)$ & & & & $<0.001$ \\
\hline Yes & $1256(18.20)$ & $497(20.60)$ & $759(17.00)$ & \\
\hline No & $5634(81.80)$ & $1920(79.40)$ & $3714(83.00)$ & \\
\hline Tumor size (\%) & & & & 0.763 \\
\hline$<2 \mathrm{~cm}$ & $4531(65.80)$ & $1607(66.50)$ & $2924(65.40)$ & \\
\hline $2-4 \mathrm{~cm}$ & $14(0.20)$ & $4(0.20)$ & $10(0.20)$ & \\
\hline$>4 \mathrm{~cm}$ & $161(2.30)$ & $57(2.40)$ & $104(2.30)$ & \\
\hline Unknown & $2184(31.70)$ & $749(31.00)$ & $1435(32.10)$ & \\
\hline Insurance status (\%) & & & & $<0.001$ \\
\hline Insured & $5268(76.50)$ & $1921(79.50)$ & $3347(74.80)$ & \\
\hline Medicaid & $1322(19.20)$ & $415(17.20)$ & $907(20.30)$ & \\
\hline Uninsured & $300(4.40)$ & $81(3.40)$ & $219(4.90)$ & \\
\hline Marital status (\%) & & & & $<0.001$ \\
\hline Married & $3311(48.10)$ & $959(39.70)$ & $2352(52.60)$ & \\
\hline Unmarried & $3579(51.90)$ & $1458(60.30)$ & $2121(47.40)$ & \\
\hline
\end{tabular}

AJCC, American Joint Committee on Cancer; SD, standard deviation; TNM, tumor, node, and metastasis.

\subsection{Survival Analysis}

\subsubsection{Sex and $O S$}

The Kaplan-Meier curves for the OS in Figure 1 shows that the survival rate varied with sex $(P<0.001)$. The median survival time was longer for female patients $(12$ months) than for male patients (9 months). Similarly, females have a higher 5-year OS ratio than males (19.2\% vs. $12.9 \%)$. In the univariate analyses, all variables were found to be significant predictors of OS. After adjustment in the multivariate analysis, the variables remained independent and significant except for race (other, $P=0.417$ ), tumor grade (IV, $P=0.671)$, and tumor size $(2-4 \mathrm{~cm}, P=0.551)$. Being male had a significant negative effect on the survival outcome (hazard ratio $[\mathrm{HR}]=1.237,95 \%$ confidence interval $[\mathrm{CI}]=1.166-1.313, P<0.001$ ) (Detailed see Table 2). 
Yaoxia Li et al:: Sex Is Related to Overall Survival in Patients with Esophageal Squamous

Cell Carcinoma: A Population-Based Study

Table 2. Cox Univariate and multivariate survival analysis of OS in patients with Esophageal squamous cell carcinoma (ESCC).

\begin{tabular}{|c|c|c|c|c|c|c|}
\hline \multirow{2}{*}{ Characteristic } & \multicolumn{3}{|c|}{ Univariate analysis } & \multicolumn{3}{|c|}{ Multivariate analysis } \\
\hline & HR & $95 \%$ CI & P-value & HR & $95 \%$ CI & P-value \\
\hline \multicolumn{7}{|l|}{ Age, years } \\
\hline$\leq 71$ & Reference & & & Reference & & \\
\hline 72-79 & 1.105 & $1.032-1.183$ & 0.004 & 1.191 & $1.111-1.278$ & $<0.001$ \\
\hline$\geq 80$ & 1.472 & $1.366-1.587$ & $<0.001$ & 1.574 & $1.454-1.704$ & $<0.001$ \\
\hline \multicolumn{7}{|l|}{ Race } \\
\hline White & Reference & & & Reference & & \\
\hline Black & 1.301 & $1.222-1.384$ & $<0.001$ & 1.140 & $1.066-1.220$ & $<0.001$ \\
\hline Other & 1.014 & $0.923-1.114$ & 0.774 & 1.040 & $0.946-1.145$ & 0.417 \\
\hline \multicolumn{7}{|l|}{ Gender } \\
\hline Female & Reference & & & Reference & & \\
\hline Male & 1.248 & $1.178-1.322$ & $<0.001$ & 1.237 & $1.166-1.313$ & $<0.001$ \\
\hline \multicolumn{7}{|c|}{ Year of diagnosis } \\
\hline $2007-2009$ & Reference & & & Reference & & \\
\hline 2010-2012 & 0.916 & $0.860-0.975$ & 0.006 & 0.931 & $0.874-0.992$ & 0.026 \\
\hline 2013-2015 & 0.882 & $0.822-0.948$ & 0.001 & 0.906 & $0.843-0.973$ & 0.007 \\
\hline \multicolumn{7}{|l|}{ Grade } \\
\hline I & Reference & & & Reference & & \\
\hline II & 1.444 & $1.269-1.644$ & $<0.001$ & 1.278 & $1.122-1.456$ & $<0.001$ \\
\hline III & 1.686 & $1.481-1.920$ & $<0.001$ & 1.347 & $1.182-1.536$ & $<0.001$ \\
\hline IV & 1.366 & $1.003-1.860$ & 0.048 & 1.069 & $0.784-1.458$ & 0.671 \\
\hline \multicolumn{7}{|c|}{ AJCC TNM stage } \\
\hline I & Reference & & & Reference & & \\
\hline II & 0.830 & $0.759-0.908$ & $<0.001$ & 0.864 & $0.790-0.946$ & 0.002 \\
\hline III & 1.138 & $1.044-1.240$ & 0.003 & 1.187 & $1.087-1.296$ & $<0.001$ \\
\hline IV & 2.198 & $2.024-2.386$ & $<0.001$ & 1.936 & $1.778-2.107$ & $<0.001$ \\
\hline \multicolumn{7}{|l|}{ Surgery } \\
\hline Yes & Reference & & & Reference & & \\
\hline No & 2.616 & $2.412-2.838$ & $<0.001$ & 1.988 & $1.827-2.164$ & $<0.001$ \\
\hline \multicolumn{7}{|l|}{ Tumor size } \\
\hline$<2 \mathrm{~cm}$ & Reference & & & Reference & & \\
\hline $2-4 \mathrm{~cm}$ & 1.099 & $0.608-1.986$ & 0.755 & 1.198 & $0.661-2.169$ & 0.551 \\
\hline$>4 \mathrm{~cm}$ & 1.435 & $1.205-1.709$ & $<0.001$ & 1.242 & $1.042-1.479$ & 0.016 \\
\hline Unknown & 1.269 & $1.198-1.345$ & $<0.001$ & 1.121 & $1.057-1.189$ & $<0.001$ \\
\hline \multicolumn{7}{|l|}{ Insurance status } \\
\hline Insured & Reference & & & Reference & & \\
\hline Medicaid & 1.220 & $1.139-1.306$ & $<0.001$ & 1.106 & $1.028-1.190$ & 0.007 \\
\hline Uninsured & 1.476 & $1.299-1.679$ & $<0.001$ & 1.348 & $1.181-1.539$ & $<0.001$ \\
\hline \multicolumn{7}{|l|}{ Marital status } \\
\hline Married & Reference & & & Reference & & \\
\hline Unmarried & 1.294 & $1.225-1.366$ & $<0.001$ & 1.206 & $1.138-1.279$ & $<0.001$ \\
\hline
\end{tabular}

HR, hazard ratio; CI, confidence interval; AJCC, American Joint Committee on cancer; TNM, tumor, node, and metastasis.

Table 3. Cox Univariate and multivariate survival analysis of patients with esophageal squamous cell carcinoma (ESCC) based on age.

\begin{tabular}{|c|c|c|c|c|c|c|c|}
\hline \multirow{2}{*}{ Characteristic } & \multirow{2}{*}{ 5-year survival, \% } & \multirow{2}{*}{ Median survival time, months } & \multicolumn{2}{|c|}{ Univariate analysis } & \multicolumn{3}{|c|}{ Multivariate analysis } \\
\hline & & & Log rank $\chi^{2}$ & P-value & HR & $95 \%$ CI & P-value \\
\hline$\leq 71$ years & & & 75.960 & $<0.001$ & & & \\
\hline Female & 23.3 & 15 & & & Reference & & \\
\hline Male & 14.4 & 9 & & & 1.330 & $1.231-1.437$ & $<0.001$ \\
\hline $72-79$ years & & & 8.193 & 0.004 & & & \\
\hline Female & 16.1 & 11 & & & Reference & & \\
\hline Male & 10.7 & 9 & & & 1.158 & $1.021-1.314$ & 0.022 \\
\hline$\geq 80$ years & & & 1.560 & 0.212 & & & \\
\hline Female & 10.9 & 6 & & & Reference & & \\
\hline Male & 7.9 & 6 & & & 1.076 & $0.931-1.243$ & 0.322 \\
\hline
\end{tabular}

HR, Full name is hazard ratio; CI, Full name is confidence interval. 


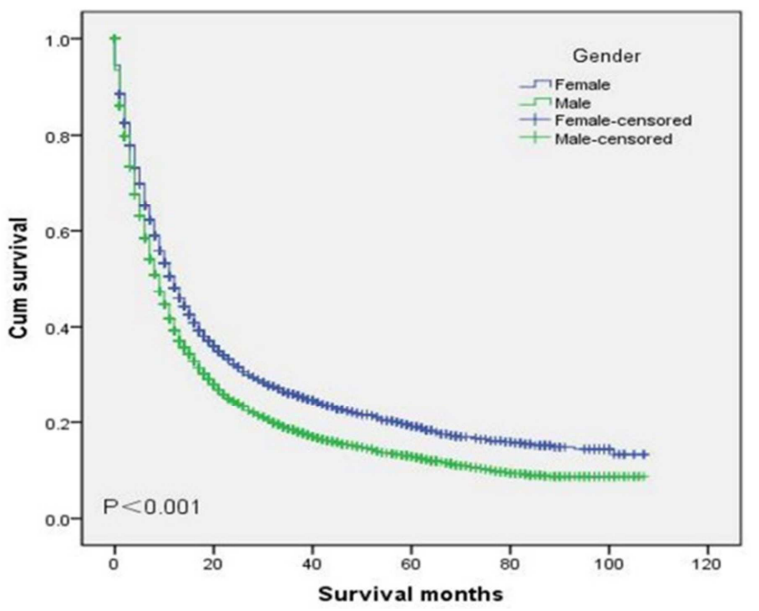

Figure 1. Kaplan-Meier (KM) survival curves of patients with esophageal squamous cell carcinoma (ESCC), SEER 2007-2015 by gender status. Cum, cumulative.

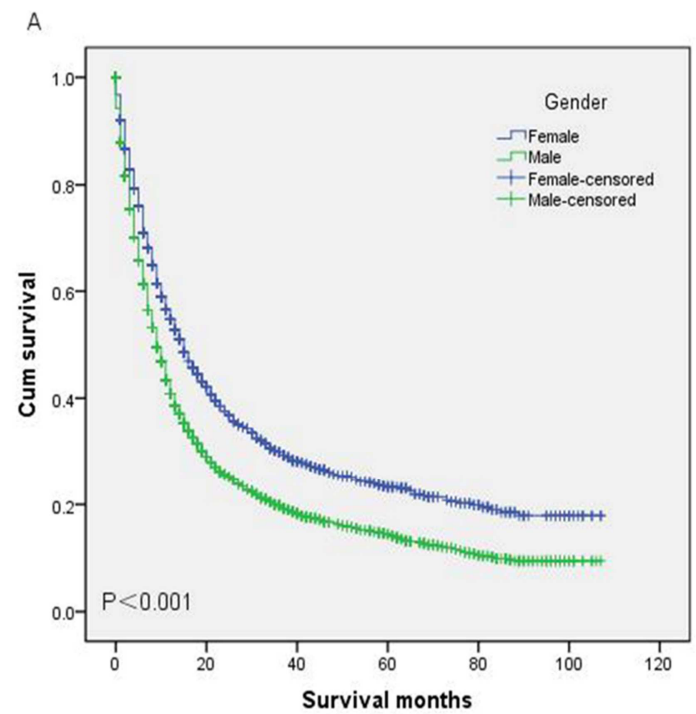

B

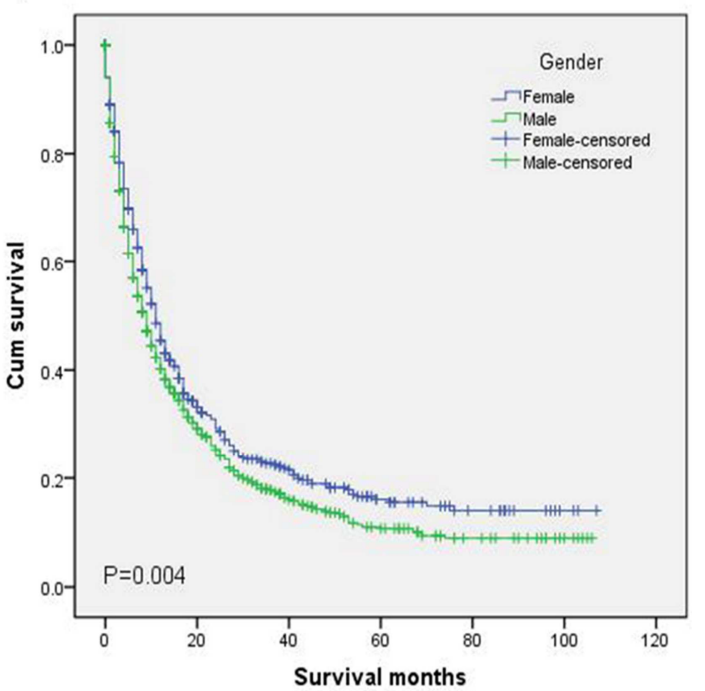

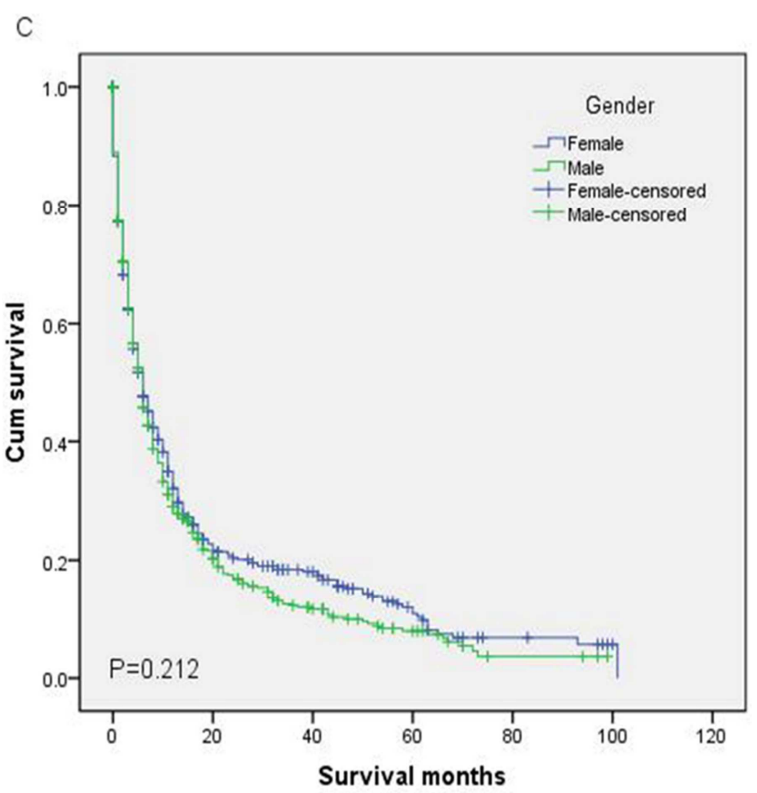

Figure 2. Kaplan-Meier (KM) Survival curves in different age subgroups of patients with esophageal squamous cell carcinoma (ESCC) according to gender status. (A) $\leq 71$ years OS. (B) $72-79$ years OS. (C) $\geq 80$ years OS. OS, overall survival; Cum, cumulative.

\subsubsection{Subgroup Analyses of the Effect of Sex According to Age}

Figure 2 illustrates the Kaplan-Meier (KM) survival curves for sex and age. The 5-year OS rate was higher in females than in males for patients aged $\leq 71$ years $(23.3 \%$ vs. $14.4 \%$, $P<0.001)$ and $72-79$ years $(16.1 \%$ vs. $10.7 \%, P=0.004)$, whereas it did not differ with sex in those aged $\geq 80$ years $(P=0.212)$. Cox Multivariate analysis showed that sex affected the OS in those patients aged $\leq 71$ years $(\mathrm{HR}=1.330,95 \%$ $\mathrm{CI}=1.231-1.437, P<0.001)$ and $72-79$ years $(\mathrm{HR}=1.158,95 \%$ $\mathrm{CI}=1.021-1.314, P=0.022$ ) (Detailed see Table 3 ).

\subsubsection{Subgroup Analyses of the Effects of Sex According to Surgery Status}

Figure 3 shows the Kaplan-Meier survival curves for sex and surgery. The 5-year OS rate was higher in female than male patients both in those who received surgery $(43.1 \%$ vs. $33.7 \%, P=0.001)$ and who did not receive surgery (13.1\% vs. $8.4 \%, P<0.001)$. Multivariate analysis showed that sex affected $\mathrm{OS}$ in both surgical recipients $(\mathrm{HR}=1.274,95 \%$ $\mathrm{CI}=1.038-1.499, \quad P=0.003)$ and non-recipient patients (HR=1.221, 95\% CI=1.146-1.302, $P<0.001$ ) (Detailed see Table 4).

\subsubsection{Subgroup Analyses of the Effect of Sex According to AJCC TNM Stage}

Figure 4 clearly shows Kaplan-Meier (KM) survival curves for male and female ESCC patients in AJCC TNM stage I $(\mathrm{n}=1166)$, stage II $(\mathrm{n}=1720)$, stage III $(\mathrm{n}=1853)$, and stage IV $(n=2151)$. The 5-year OS rate was higher in females than in males for patients at AJCC TNM stage II (29.2\% vs. $20.2 \%$, $P<0.001)$, stage III (18.9\% vs. $14.0 \%, P=0.016)$, and stage IV (5.2\% vs. $3.0 \%, P<0.001)$, while there was no sex-related 
difference for stage I. Cox multivariate analysis indicated that sex was a significant independent prognostic factor for OS in ESCC patients with different AJCC TNM stages, not only at stage I $(\mathrm{HR}=1.202,95 \% \mathrm{CI}=1.040-1.388, P=0.013)$, stage II

A

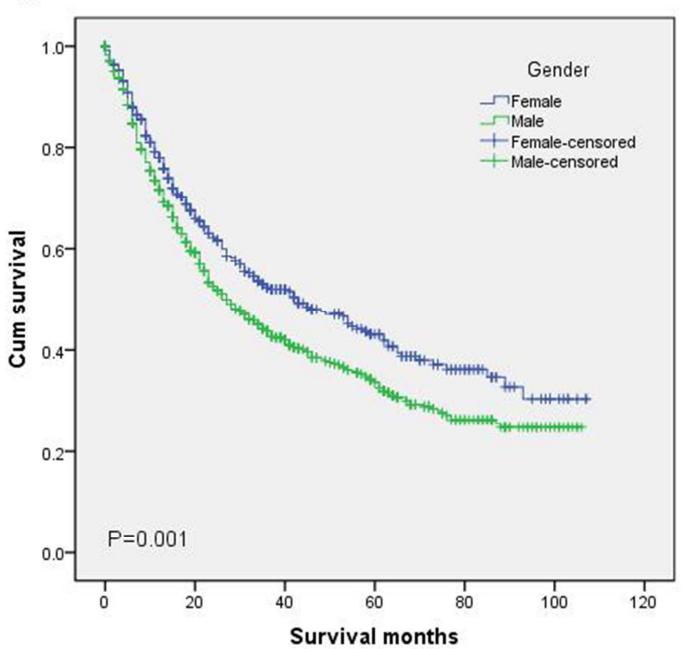

$(\mathrm{HR}=1.337,95 \% \mathrm{CI}=1.179-1.516, P<0.001)$, but also at stage III $(\mathrm{HR}=1.156,95 \% \mathrm{CI}=1.030-1.298, P=0.014)$, and stage IV $(\mathrm{HR}=1.268,95 \% \mathrm{CI}=1.145-1.404, P<0.001)$ (Detailed see Table 5).

B

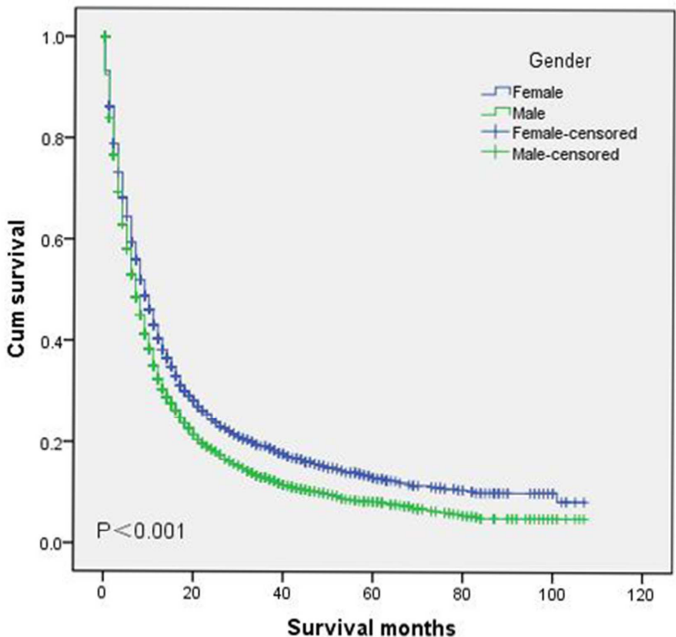

Figure 3. Survival curves in different surgery subgroups of patients with esophageal squamous cell carcinoma according to gender status. (A) Surgery OS. (B) No surgery OS. OS, overall survival; Cum, cumulative.

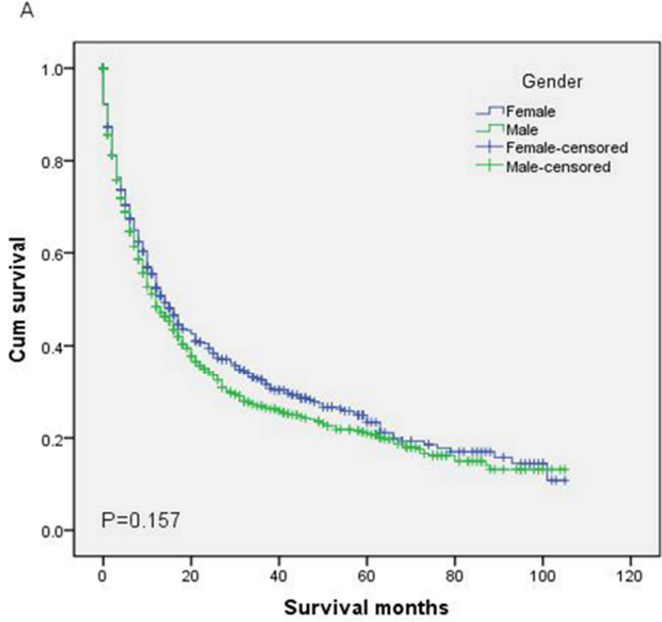

$c$

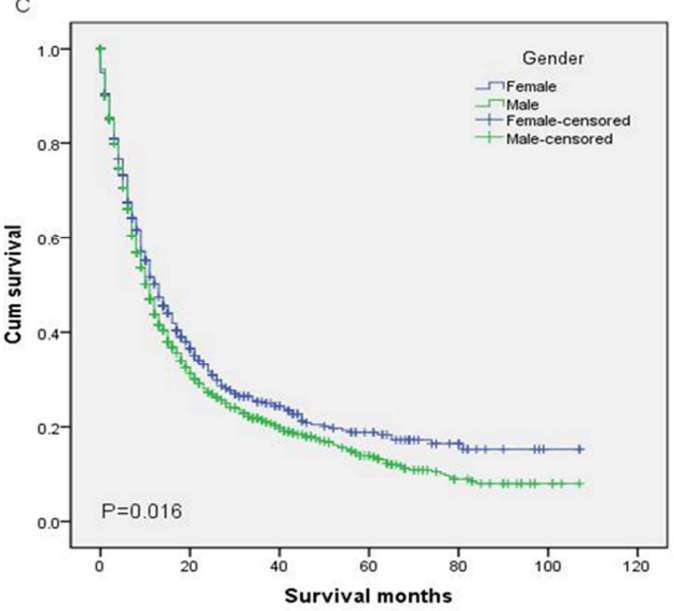

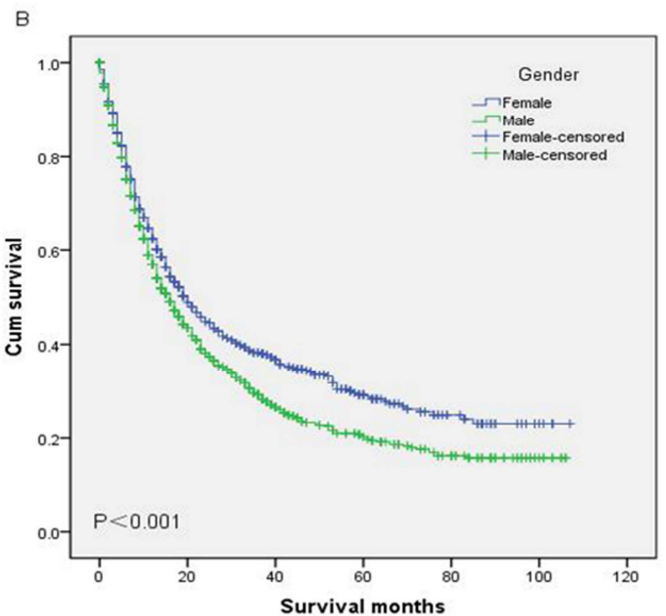

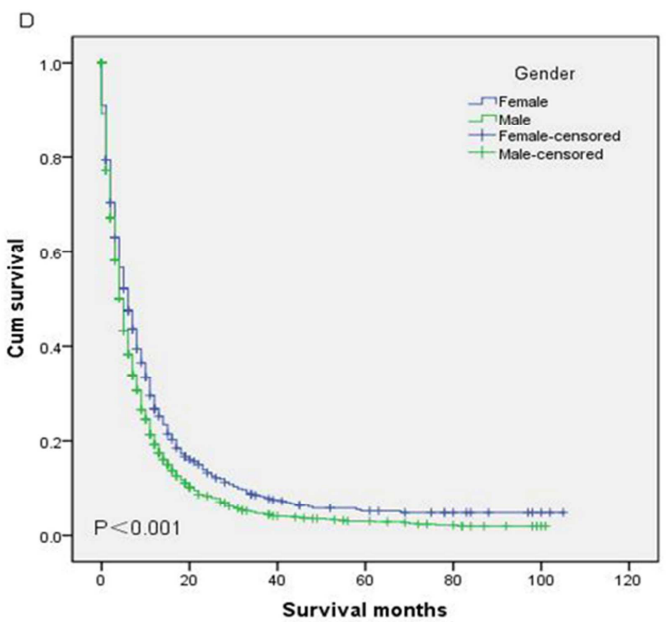

Figure 4. According to different gender status, survival curves of ESCC patients with different tumor Tumor-Node-Metastasis stage subgroups were obtained by survival analysis. (A) Stage I OS. (B) Stage II OS. (C) Stage III OS. (D) Stage IV OS. OS, overall survival; Cum, cumulative. 
Table 4. Univariate and multivariate survival analysis of patients with esophageal squamous cell carcinoma (ESCC) according to surgery details.

\begin{tabular}{|c|c|c|c|c|c|c|c|}
\hline \multirow{2}{*}{ Characteristic } & \multirow{2}{*}{ 5-year survival, \% } & \multirow{2}{*}{ Median survival time, months } & \multicolumn{2}{|c|}{ Univariate analysis } & \multicolumn{3}{|c|}{ Multivariate analysis } \\
\hline & & & Log $\operatorname{rank} \chi_{2}$ & P-value & HR & $95 \% \mathrm{CI}$ & P-value \\
\hline Surgery & & & 10.127 & 0.001 & & & \\
\hline Female & 43,1 & 43 & & & Reference & & \\
\hline Male & 33.7 & 27 & & & 1.274 & $1.038-1.499$ & 0.003 \\
\hline No surgery & & & 40.176 & $<0.001$ & & & \\
\hline Female & 13.1 & 9 & & & Reference & & \\
\hline Male & 8.4 & 7 & & & 1.221 & $1.146-1.302$ & $<0.001$ \\
\hline
\end{tabular}

HR, Full name is hazard ratio; CI, Full name is confidence interval.

Table 5. Cox regression univariate and multivariate survival analyses of patients with esophageal squamous cell carcinoma (ESCC) according to the Cancer Tumor- Node-Metastasis stage of American Joint Committee.

\begin{tabular}{|c|c|c|c|c|c|c|c|}
\hline \multirow[b]{2}{*}{ Characteristic } & \multirow[b]{2}{*}{ 5-year survival, \% } & \multirow[b]{2}{*}{ Median survival time, months } & \multicolumn{2}{|c|}{ Univariate analysis } & \multicolumn{3}{|c|}{ Multivariate analysis } \\
\hline & & & Log rank $\chi_{2}$ & P-value & HR & $95 \%$ CI & P-value \\
\hline & & & 2.005 & 0.157 & & & \\
\hline Female & 23.4 & 14 & & & Reference & & \\
\hline Male & 21.0 & 12 & & & 1.202 & $1.040-1.388$ & 0.013 \\
\hline II & & & 12.175 & $<0.001$ & & & \\
\hline Female & 29.2 & 20 & & & Reference & & \\
\hline Male & 20.2 & 16 & & & 1.337 & $1.179-1.516$ & $<0.001$ \\
\hline III & & & 5.825 & 0.016 & & & \\
\hline Female & N/A & 13 & & & Reference & & \\
\hline Male & 14.0 & 11 & & & 1.156 & $1.030-1.298$ & 0.014 \\
\hline IV & & & 17.844 & $<0.001$ & & & \\
\hline Female & 5.2 & 6 & & & Reference & & \\
\hline Male & 3.0 & 5 & & & 1.268 & $1.145-1.404$ & $<0.001$ \\
\hline
\end{tabular}

$\mathrm{HR}$, Full name is hazard ratio; CI, Full name is confidence interval.

\section{Discussion}

This study investigated the relationship between sex and OS in patients with ESCC in the United States. Univariate and multivariate analyses revealed that the incidence of OS was higher in female patients than in male patients, suggesting that sex is an important independent prognostic factor for the survival of patients with ESCC. The results of our research are similar to those of previous studies that analyzed data in the SEER database to explore the relationship between sex and survival in patients with liver cancer [16]. Westwick et al. found that the mortality rate of patients with spinal meningioma was higher in males than in females, which also supports the results of the present study [22]. The sex-related difference in survival rate might be related to differences in hormone levels, since estrogen and progesterone in females can reportedly reduce their risk of developing ESCC [23], and overexpression of the androgen receptor is related to the occurrence of ESCC and adenocarcinoma [24].

The prognosis of cancer patients differs significantly with age $[25,26]$, and so we performed subgroup analyses of the effects of sex on patients with ESCC at different ages. The results showed that among patients with ESCC aged $\leq 71$ and 72-79 years, females had a significant survival advantage over males, whereas the OS did not differ between male and female patients aged $\geq 80$ years. The sex-related difference in survival rate among patients aged $\leq 79$ years may be due to better compliance behaviors of females compared with males [27], while patients aged 70-80 years have lower tumor invasiveness, resulting in prognoses that are more similar to those of young people [28]. However, both female and male patients aged $\geq 80$ years were found to have significantly increased mortality due to their poor functional status [29], which could explain the absence of a difference in OS between males and females in that age group.

Some previous studies have shown that surgical resection is associated with better survival in patients with ESCC [7], whereas Chang et al. reported that treatments such as surgery do not affect the OS of these patients [30]. These two contrasting findings explain why we conducted subgroup analyses of the effects of surgery on sex. Our results show that sex is not only an independent prognostic factor for patients who receive surgery with OS, but also an independent prognostic factor for those who do not receive surgery. Therefore, sex is a protective factor for both ESCC patients who have received surgery and those who have not, which should be paid attention to.

Zhong et al. found that AJCC TNM stages II and III were related to the survival of ESCC patients, which may be closely related to lymph node dissection [31]. However, the subgroup analyses of AJCC TNM staging performed in the present study showed that sex is an independent prognostic factor for OS in patients at all four stages (I, II, III, and IV). Therefore, sex is a protective factor not only for patients with mid-term ESCC, but also for those with early and late ESCC, and its influence can therefore not be ignored.

Despite insurance status being closely related to the prognosis of patients [32], most previous studies have not included insurance status when investigating the factors that affect the prognosis of ESCC [33]. Therefore, the present 
study included insurance status in order to more-comprehensively analyze the important factors that affect the prognosis of ESCC patients. This study found that insurance status is a meaningful independent prognostic factor for the survival of patients with ESCC. Compared with insured patients, the risk of death is higher for Medicaid patients $(\mathrm{HR}=1.106,95 \% \mathrm{CI}=1.028-1.190, P=0.007)$ and uninsured patients $(\mathrm{HR}=1.348, \quad 95 \% \quad \mathrm{CI}=1.181-1.539$, $P<0.001)$. These findings might be due to the socioeconomic status being better for patients who purchase insurance than for uninsured patients, and they are consistent with the findings of Wang et al. [19] and Perry et al. [32].

To the best of our knowledge, the present research is the first population-based research to explore the association between sex and OS in patients with ESCC. Afshar et al. found that female patients have a higher risk of death and a lower survival rate than male patients with bladder cancer, renal pelvis cancer, and ureteral cancer; however, that study did not include patients with ESCC [17]. Our results showed that in a cohort of patients with ESCC, the survival time was significantly longer and the OS rate was significantly higher in females than in males.

There are multiple possible factors underlying why female ESCC patients show better survival. A much lower proportion of female patients than male patients drink alcohol and smoke [34, 35], and both drinking and smoking increase the risk of ESCC [36]. Smoking can cause oxidative stress and DNA damage in the body, and cigarettes release carcinogens such as nitrosamines and damaging aromatic hydrocarbons during combustion, which increase the risk of esophageal cancer [37, 38]. Drinking alcohol can inhibit the activity of natural killer cells and promote tumor metastasis [39]. In addition, tobacco and alcohol can produce focal disturbances in the expression of $27-\mathrm{kDa}$ heat shock protein in the esophagus, which can promote the occurrence of ESCC [40]. Male patients, with their higher rates of smoking and drinking alcohol [41], will potentially be more affected by lasting damage to the esophagus, which will affect the effectiveness of treatment and prognosis to varying degrees. These reasons can explain why the OS rate of male patients with ESCC is lower than that of female patients with ESCC.

We also found that the proportion of patients receiving surgery was higher among females than males. The surgery status is an important factor for improving the survival time of patients with ESCC [3, 7], since surgery can involve removing tumors from primary or distant metastatic sites, and performing lymph node dissection. Haisley et al. found that each $1-\mathrm{cm}$ increase in tumor length decreasing $\mathrm{OS}(\mathrm{HR}=1.18,95 \% \mathrm{CI}=1.06-1.32, P=0.003)$ [42]. Surgical resection can also inhibit tumor progression [3, 7]. In addition, studies have shown that the survival advantage of female cancer patients over male cancer patients is closely related to their higher treatment compliance and better health behaviors [27], while a lower acceptance rate of surgery, treatment compliance, and healthy behaviors reduce the survival potential of male patients $[3,7,27]$. Our results suggest that it is not enough to focus on differences in cancer survival at the levels of age, surgery, radiotherapy, and chemotherapy; instead, there is an urgent need to also pay attention to sex differences in survival among patients with ESCC. Early screening, guidance, and assistance are needed for improving the OS of high-risk male patients with ESCC.

Some limitations of the current research should be considered. First, the SEER data registry has limitations in providing information about patients and does not report any comorbidities or complications [43]. Second, the SEER database temporarily not provided specific information about radiotherapy, chemotherapy, or drug use [18], and these variables will certainly affect the prognosis of patients with ESCC. Future studies should attempt to adjust for these covariates in order to obtain more-comprehensive results. Third, the income and education levels of individual patents are not available in the SEER database, and both of these factors may affect treatment choices [19]. Fourth, the SEER database does not record poor habits in the daily lives of patients, such as drinking alcohol and smoking. These two factors are closely related to the prognosis of patients with ESCC [44], but we could not include them in this study. Finally, our study had a short follow-up, which was due to data on the insurance status only being added to the SEER database in 2007, and the data only being available up to 2015 . Longer follow-up periods are needed in future studies to verify whether the present results also apply over longer time periods.

Notwithstanding the aforementioned potential limitations, the present findings suggest that being female is associated with better survival in patients with ESCC, independent of the other analyzed variables. Our results clearly indicate that particular focus needs to be placed on male patients diagnosed with ESCC, and prospective prognostic interventions and health education should be applied to improve their survival. In addition, early and active screening is recommended for males with other risk factors for ESCC.

\section{Conclusion}

Female ESCC patients have significantly better OS than men. Sex is an independent prognostic factor for ESCC.

Currently, it is generally accepted that female patients with ESCC have a better survival rate than male patients because the proportion of drinking and smoking among female patients is much lower than that of male patients, which reduces the risk of female patients with ESCC to some extent and is more conducive to their recovery after the disease.

Women's better treatment compliance and health behaviors, as well as their higher surgical acceptance rate, are important reasons for the higher survival rate of women with ESCC than men.

Prospective intervention measures and health education for male ESCC patients are particularly important to reduce their mortality and improve their prognosis.

\section{Current Research Questions}

What are the barriers to early screening and intervention for male ESCC patients?

How to implement early intervention to reduce the mortality rate of male ESCC patients?

Can early comprehensive intervention effectively improve the prognosis of patients with advanced ESCC? 


\section{Contributorship Statement}

Qiao-hong Yang: Conceptualization, Methodology, Project administration. Yao-xia $\mathrm{Li}$ and $\mathrm{Na}$ Wang: Software, Data curation, Writing-Original draft preparation. Jia-le Wang and Jun Lyu: Visualization, Supervision, Investigation. Zhuo-rui Zhao, Qin-yang Wu and Qi-qi Ke: Supervision, WritingReviewing and Editing.

\section{Competing Interests}

All the authors do not have any possible conflicts of interest.

\section{References}

[1] Global Burden of Disease Cancer C, Fitzmaurice C, Allen C, et al. Global, Regional, and National Cancer Incidence, Mortality, Years of Life Lost, Years Lived With Disability, and Disability-Adjusted Life-years for 32 Cancer Groups, 1990 to 2015: A Systematic Analysis for the Global Burden of Disease Study. JAMA Oncol 2017; 3 (4): 524-48 doi: 10.1001/jamaoncol.2016.5688 [published Online First: 2016/12/06].

[2] Siegel RL, Miller KD, Jemal A. Cancer statistics, 2019. CA Cancer J Clin 2019; 69 (1): 7-34 doi: 10.3322/caac.21551 [published Online First: 2019/01/09].

[3] Then EO, Lopez M, Saleem S, et al. Esophageal Cancer: An Updated Surveillance Epidemiology and End Results Database Analysis. World J Oncol 2020; 11 (2): 55-64 doi: 10.14740/wjon1254 [published Online First: 2020/04/15].

[4] Napier KJ, Scheerer M, Misra S. Esophageal cancer: A Review of epidemiology, pathogenesis, staging workup and treatment modalities. World J Gastrointest Oncol 2014; 6 (5): 112-20 doi: 10.4251/wjgo.v6.i5.112 [published Online First: 2014/05/17].

[5] Pennathur A, Luketich JD. Resection for esophageal cancer: strategies for optimal management. Ann Thorac Surg 2008; 85 (2): S751-6 doi: 10.1016/j.athoracsur.2007.11.078 [published Online First: 2008/01/29].

[6] Qiu W, Chen J, Huang X, et al. The analysis of the lipid levels in patients with coronary artery disease after percutaneous coronary intervention: a one-year follow-up observational study. Lipids Health Dis 2020; 19 (1): 163 doi: 10.1186/s12944-020-01340-5 [published Online First: 2020/07/08].

[7] Worni M, Martin J, Gloor B, et al. Does surgery improve outcomes for esophageal squamous cell carcinoma? An analysis using the surveillance epidemiology and end results registry from 1998 to 2008. J Am Coll Surg 2012; 215 (5): 643-51 doi: 10.1016/j.jamcollsurg.2012.07.006 [published Online First: 2012/10/23].

[8] Worni M, Castleberry AW, Gloor B, et al. Trends and outcomes in the use of surgery and radiation for the treatment of locally advanced esophageal cancer: a propensity score adjusted analysis of the surveillance, epidemiology, and end results registry from 1998 to 2008. Dis Esophagus 2014; 27 (7): 662-9 doi: 10.1111/dote.12123 [published Online First: 2013/08/14].

[9] Sjoquist KM, Burmeister BH, Smithers BM, et al. Survival after neoadjuvant chemotherapy or chemoradiotherapy for resectable oesophageal carcinoma: an updated meta-analysis.
The Lancet Oncology 2011; 12 (7): 681-92 doi: 10.1016/s1470-2045(11)70142-5.

[10] Kauppila JH, Wahlin K, Lagergren P, et al. Sex differences in the prognosis after surgery for esophageal squamous cell carcinoma and adenocarcinoma. Int J Cancer 2019; 144 (6): 1284-91 doi: 10.1002/ijc.31840 [published Online First: 2018/09/01].

[11] Gavin AT, Francisci S, Foschi R, et al. Oesophageal cancer survival in Europe: a EUROCARE-4 study. Cancer Epidemiol 2012; 36 (6): 505-12 doi: 10.1016/j.canep.2012.07.009 [published Online First: 2012/08/23].

[12] Eder L, Thavaneswaran A, Chandran V, et al. Gender difference in disease expression, radiographic damage and disability among patients with psoriatic arthritis. Ann Rheum

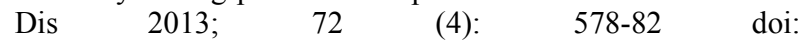
10.1136/annrheumdis-2012-201357 [published Online First: 2012/05/17].

[13] Fajkovic H, Halpern JA, Cha EK, et al. Impact of gender on bladder cancer incidence, staging, and prognosis. World J Urol 2011; 29 (4): 457-63 doi: 10.1007/s00345-011-0709-9 [published Online First: 2011/06/10].

[14] Sundermann E E, Biegon A, Rubin L H, et al. Better verbal memory in women than men in MCI despite similar levels of hippocampal atrophy. Neurology 2016; 86 (15): 1368-1376 doi: 10.1212/WNL.0000000000002570 [published Online First: 2016/03/16].

[15] Nebel RA, Aggarwal NT, Barnes LL, et al. Understanding the impact of sex and gender in Alzheimer's disease: A call to action. Alzheimers Dement 2018; 14 (9): 1171-83 doi: 10.1016/j.jalz.2018.04.008 [published Online First: 2018/06/17].

[16] Zhang W, Jin K, Wang F, et al. Differences in the prognostic value of tumor size on hepatocellular cancer-specific survival stratified by gender in a SEER population-based study. United European Gastroenterol J 2019; 7 (7): $933-41$ doi: 10.1177/2050640619845602 [published Online First: 2019/08/21].

[17] Afshar N, English DR, Thursfield V, et al. Differences in cancer survival by sex: a population-based study using cancer registry data. Cancer Causes Control 2018; 29 (11): 1059-69 doi: 10.1007/s10552-018-1079-z [published Online First: 2018/09/09].

[18] Tramontano AC, Nipp R, Mercaldo ND, et al. Survival Disparities by Race and Ethnicity in Early Esophageal Cancer. Dig Dis Sci 2018; 63 (11): 2880-88 doi: 10.1007/s10620-018-5238-6 [published Online First: 2018/08/16].

[19] Wang N, Bu Q, Yang J, et al. Insurance status is related to overall survival in patients with small intestine adenocarcinoma: A population-based study. Curr Probl Cancer 2020; $44 \quad$ (1): 400505 doi: 10.1016/j.currproblcancer.2019.100505 [published Online First: 2019/09/25].

[20] Doll KM, Rademaker A, Sosa JA. Practical Guide to Surgical Data Sets: Surveillance, Epidemiology, and End Results (SEER) Database. JAMA Surg 2018; 153 (6): 588-89 doi: 10.1001/jamasurg.2018.0501 [published Online First: 2018/04/05].

[21] You H, Yang J, Liu Q, et al. The impact of the lymph node density on overall survival in patients with Wilms' tumor: a SEER analysis. Cancer Manag Res 2018; 10: 671-77 doi: 10.2147/CMAR.S163514 [published Online First: 2018/04/20]. 
[22] Westwick HJ, Shamji MF. Effects of sex on the incidence and prognosis of spinal meningiomas: a Surveillance, Epidemiology, and End Results study. J Neurosurg Spine 2015; 23 (3): 368-73 doi: 10.3171/2014.12.SPINE14974 [published Online First: 2015/05/30].

[23] Brusselaers N, Maret-Ouda J, Konings P, et al. Menopausal hormone therapy and the risk of esophageal and gastric cancer. Int J Cancer 2017; 140 (7): 1693-99 doi: 10.1002/ijc.30588 [published Online First: 2016/12/23].

[24] Sukocheva OA, Li B, Due SL, et al. Androgens and esophageal cancer: What do we know? World J Gastroenterol 2015; 21 (20): 6146-56 doi: 10.3748/wjg.v21.i20.6146 [published Online First: 2015/06/03].

[25] Nipp R, Tramontano AC, Kong CY, et al. Disparities in cancer outcomes across age, sex, and race/ethnicity among patients with pancreatic cancer. Cancer Med 2018; 7 (2): 525-35 doi: 10.1002/cam4.1277 [published Online First: 2018/01/13].

[26] Zeng C, Wen W, Morgans AK, et al. Disparities by Race, Age, and Sex in the Improvement of Survival for Major Cancers: Results From the National Cancer Institute Surveillance, Epidemiology, and End Results (SEER) Program in the United States, 1990 to 2010 . JAMA Oncol 2015; 1 (1): 88-96 doi: 10.1001/jamaoncol.2014.161 [published Online First: 2015/07/17].

[27] Innos K, Padrik P, Valvere V, et al. Sex differences in cancer survival in Estonia: a population-based study. BMC Cancer 2015; 15: 72 doi: 10.1186/s12885-015-1080-9 [published Online First: 2015/04/18].

[28] Hambraeus G M, Mercke C E, Willen R, et al. Prognostic factors influencing survival in combined radiotherapy and surgery of squamous cell carcinoma of the esophagus with special reference to a histopathologic grading system. Cancer 1988; $\quad 62 \quad$ (5): $\quad 895-904 \quad$ doi: 10.1002/1097-0142(19880901)62:5<895::aid-cncr282062050 8>3.0.co;2-0. [published Online First: 1988/03/02]

[29] Yang HX, Ling L, Zhang X, et al. Outcome of elderly patients with oesophageal squamous cell carcinoma after surgery. Br J Surg 2010; 97 (6): 862-7 doi: 10.1002/bjs.7005 [published Online First: 2010/03/20].

[30] Chang DT, Chapman C, Shen J, et al. Treatment of esophageal cancer based on histology: a surveillance epidemiology and end results analysis. Am J Clin Oncol 2009; 32 (4): 405-10 doi: 10.1097/COC.0b013e3181917158 [published Online First: 2009/05/06].

[31] Haberlin C, O'Dwyer T, Mockler D, et al. The use of eHealth to promote physical activity in cancer survivors: a systematic review. Support Care Cancer 2018; 26 (10): 3323-36 doi: 10.1007/s00520-018-4305-z [published Online First: 2018/06/18].

[32] Perry AM, Brunner AM, Zou T, et al. Association between insurance status at diagnosis and overall survival in chronic myeloid leukemia: A population-based study. Cancer 2017; 123 (13): 2561-69 doi: 10.1002/cncr.30639 [published Online First: 2017/05/04].
[33] Zhang J, Jiang Y, Wu C, et al. Comparison of clinicopathologic features and survival between eastern and western population with esophageal squamous cell carcinoma. J Thorac Dis 2015; 7 (10): 1780-6 doi: 10.3978/j.issn.2072-1439.2015.10.39 [published Online First: 2015/12/02].

[34] Padrao P, Damasceno A, Silva-Matos C, et al. Alcohol consumption in Mozambique: regular consumption, weekly pattern and binge drinking. Drug Alcohol Depend 2011; 115 (1-2): 87-93 doi: 10.1016/j.drugalcdep.2010.10.010 [published Online First: 2010/12/03].

[35] Soler Vila H, Galán I, Valencia Martín J L, et al. Binge Drinking in Spain, 2008-2010. Alcoholism: Clinical and Experimental Research 2014; 38 (3): $810-819$ doi: 10.1111/acer.12275 [published Online First: 2013/10/24].

[36] Freedman ND, Abnet CC, Leitzmann MF, et al. A prospective study of tobacco, alcohol, and the risk of esophageal and gastric cancer subtypes. Am J Epidemiol 2007; 165 (12): 1424-33 doi: 10.1093/aje/kwm051 [published Online First: 2007/04/11].

[37] Mena S, Ortega A, Estrela JM. Oxidative stress in environmental-induced carcinogenesis. Mutat Res 2009; 674 (1-2): 36-44 doi: 10.1016/j.mrgentox.2008.09.017 [published Online First: 2008/11/04].

[38] Hecht SS. Progress and challenges in selected areas of tobacco carcinogenesis. Chem Res Toxicol 2008; 21 (1): 160-71 doi: 10.1021/tx7002068 [published Online First: 2007/12/07].

[39] Ben-Eliyahu S, Page GG, Yirmiya R, Taylor AN. Acute alcohol intoxication suppresses natural killer cell activity and promotes tumor metastasis. Nat Med. 1996, 2 (4): 457-460 doi: 10.1038/nm0496-457 [published Online First: 1996/04/01].

[40] Lambot M A, Peny M O, Fayt I, et al. Overexpression of $27-\mathrm{kDa}$ heat shock protein relates to poor histological differentiation in human oesophageal squamous cell carcinoma. Histopathology. 2000; 36 (4): 326-330 doi: 10.1046/j.1365-2559.2000.00858.x [published Online First: 2001/12/25].

[41] Hidaka H, Hotokezaka M, Nakashima S, et al. Sex difference in survival of patients treated by surgical resection for esophageal cancer. World J Surg 2007; 31 (10): $1982-7$ doi: 10.1007/s00268-007-9193-1 [published Online First: 2007/08/07].

[42] Haisley KR, Hart KD, Fischer LE, et al. Increasing tumor length is associated with regional lymph node metastases and decreased survival in esophageal cancer. Am J Surg 2016; 211 (5): 860-6 doi: 10.1016/j.amjsurg.2016.01.007 [published Online First: 2016/03/20].

[43] Arce PM, Camilon PR, Stokes WA, et al. Is sex an independent prognostic factor in cutaneous head and neck melanoma? Laryngoscope 2014; 124 (6): 1363-7 doi: 10.1002/lary.24439 [published Online First: 2013/10/15].

[44] Xie SH, Lagergren J. Risk factors for oesophageal cancer. Best Pract Res Clin Gastroenterol 2018; 36-37: 3-8 doi: 10.1016/j.bpg.2018.11.008 [published Online First: 2018/12/16]. 